Received: 2016-10-13

Revised: 2016-11-12

Accepted: 2016-11-14

\title{
A Cohort Study Protocol of Low Back Pain in Rural Area Inhabitants: Fasa Low Back Pain Cohort Study (FABPACS)
}

Mojtaba Farjam ${ }^{1,2}$, Alireza Askari ${ }^{3}$, Ali Hoseinipour ${ }^{4}$, Reza Homayounfar ${ }^{1}$, Javad Jamshidi ${ }^{1}$, Fatemeh Khodabakhshi ${ }^{5}$, Habib Zakeri ${ }^{1,4 \bowtie}$

'Noncommunicable Diseases Research Center, Fasa University of Medical Sciences, Fasa, Iran

${ }^{2}$ Department of Medical Pharmacology, Fasa University of Medical Sciences, Fasa, Iran

${ }^{3}$ Department of Orthopedic Surgery, School of Medicine, Shiraz University of Medical Sciences, Shiraz, Iran

${ }^{4}$ Department of Anesthesiology, Fasa University of Medical Sciences, Fasa, Iran

${ }^{5}$ Student Research Committee, Fasa University of Medical Sciences, Fasa, Iran

\begin{abstract}
Background: Low back pain (LBP) is one of the main causes of disability in most societies that imposes much cost to the economic and health systems. In Iranian population, a prevalence as high as $27 \%$ has been reported for chronic LBP. So this study was designed to investigate the factors associated with low back pain in Iranian population. Methods/ Design: In Fasa Cohort Study, a branch of Persian cohort study, LBP patients were registered among the participants. A total of 10000 peoples, 1700 patients enrolled in cohort study were registered as LBP patients. In addition to detailed demographic, socioeconomic, anthropometric, nutrition, and medical history, limited physical examinations, determination of physical activity and body composition that was obtained in the cohort study, history of LBP, assessment of the pain severity, McGill pain inventory, and Oswestry questionnaire was filled for the LBP patients. All data are stored online using a dedicated software. Discussion: The cohort study is the best way to collect the necessary information required for policy making in the field of LBP. This study will help in providing some information about LBP in our area to establish a better management of the disease. Moreover, this study will provide many opportunities for clinical trials in this field, and we are going to do interventional studies in the cohort in future.[GMJ.2016;5(4):225-29]
\end{abstract}

Keywords: Low Back Pain; Cohort Study; Rural Area; Protocol; Fasa.

\section{Introduction}

Tow back pain (LBP) is a common and frequently debilitating health event compromising a high referral rate to therapeutic health care systems and a wide range of severity [1]. Worldwide, the lifetime incidence of LBP is very high [2], and it is believed that LBP is the first cause for Years Lived with Disability (YLDs). The burdens of this health problem in low to moderate- income societ-

\section{GMJ}

2016 Galen Medical Journal

Tel/Fax: +98 7136474503

PO Box 7193616563

Email:info@gmj.ir ies are inevitably high, and health systems have to invest in preventive and rehabilitation methods, however, this could be carried out following elucidation of risk factors in every country [3].

In Iranian population, based on a cross- sectional study, a prevalence of chronic LBP of more than $27 \%$ has been reported [4]. The disease has been attracting attention in Iran recently and deserved attention [5].

\footnotetext{
Correspondence to:

Habib Zakeri, Noncommunicable Diseases Research Center, Fasa University of Medical Sciences, Fasa, Iran Telephone Number: +98 7153316300

Email Address : zakerihabib@gmail.com
} 
To providing preventive care for LBP, risk factor analyses have been performed in many countries. Several cohort studies have been designed to elucidate the risk factors and the outcomes of LBP, with the ultimate hope to tailor evidence- based interventions to prevent the disease in all levels of prevention [2, 6-10]. However, in Iran, a comprehensive cohort study to assess the LBP disease in a context of detailed health evaluation is not available in the literature. This evaluation is of extreme importance in a wide range of comorbidities, and so synergistic burdens have been attributed to LBP [11].

\section{Methods/Design}

\section{Participant Recruitment}

In Fasa Cohort Study [12], a branch of Persian cohort study, LBP patients were registered among the participants. All people in this cohort study are 10000 men and women between the ages of 35 to 70 years old living in rural areas around Fasa city centered by Sheshdeh. Evaluation of non-communicable diseases in this population as the target and a longitudinal follow-up of 15 years was planned. The enrolment of the people was done by the help of Behvarzes working in health houses in every village. Behvarzes are the health workers in Iranian rural societies, each one covering health for 200 to 2000 people with close observation [13]. For cohort study, they were trained to invite their attributed population according to a time- table.

\section{Target Region}

Sheshdeh (28 $\left.56^{\prime} 56.0^{\prime \prime} \mathrm{N} 53^{\circ} 59^{\prime} 26.9^{\prime \prime} \mathrm{E}\right)$ with a total population of 41000 is located in the territory of Fasa University of Medical Sciences. This region was selected as the cohort area. Fasa city is a mid- class urban/ rural area in the southeast of the state of Fars which is located in the southwest of Iran.

Details regarding this cohort region and the steps of work have been reported in the study protocol of Fasa cohort study [12]. This study is based on Persian cohort study, approved by deputy of research and technology of the Iranian ministry of health. Briefly, a field office for this study was established in Sheshdeh.
This area office contained a reception desk and several rooms for taking history, physical examination, and biological sampling by a field team including physicians, nurses, lab technicians and interviewers working daily. The registration is done by giving an 11- digits code to each individuals after taking an informed consent letter. The interviews are actively performed by trained general, medical and nutrition professionals, in separate rooms. The sampling process is carried out in the sampling room. The sample tests and savings were conducted in the central laboratory of Fasa University. The details of histories taken, nutrition interviews, anthropometric evaluations and laboratory samples are according to previous study [12].

\section{Assessment of LBP}

All patients are asked about the history of LBP. This question is represented in the medical interview and the exact translation of the question is:

"Have you ever had a low back pain that lasts more than one week, in a way that it had disrupted your activities of daily living?"

For those individuals with a negative history of LBP until the time point of cohort recruitment, the routine track of interviews continues. For people with a positive history of LBP, a new corridor is opened to LBP evaluation section. They are guided to this room after all steps fulfill.

\section{LBP Evaluation Section}

To assessment the quality and quantity of LBP at the first interface of the individual with the cohort, a basic evaluation is carried out in this section as noted below. The whole process is performed under the supervision of pain/ anesthesiology specialist physicians.

Non-specific LBP including different self- reported presentations as tension, soreness and/ or stiffness in the lower back region for which finding a specific cause is impossible. Various structures in the back, such as the discs, the joints, and connective tissues may contribute to symptoms.

The lower back is anatomically the area between the bottom of the rib cage and the buttock creases. Some LBP patients might 
complain of pain in their upper regions of lower extremities including proximal of legs although the pain mostly is more sensed in the back region as defined above.

The anesthesiology medical doctor is responsible for ruling out specific causes of LBP (such as malignancy, infection, fracture, ankylosing spondylitis and other inflammatory disorders). Specific causes of LBP are the exclusion criteria in this study. Pregnant women with LBP are also excluded.

Target outcomes are chosen by some core domains for LBP according to the latest findings recommended.

Many suspected variables and factors are evaluated in the project uncluding: The LBP and associated factors, pain intensity spectrum in LBP, the relationship between back pain with occupational injuries, high-risk behavior about chronic LBP, determination of survival and disability in chronic low back pain, co-existing disease with LBP, the incidence of cardiovascular disease in LBP patients, prevalence of surgery related to LBP, incidence of LBP after surgery, prevalence of psychiatric disorders, overweight and obesity, smoking, sleep, physical activity, nutritional data, socioeconomic and lifestyle factors, back-specific function biological factors and etc.

\section{History of LBP}

Trained anesthesiology nurses record all the details regarding any event of pain. These include the time and duration of LBP, the medical referrals, and paramedical evaluations are recorded. If patients do not remember their LBP history details, his/ her name is written and the documents will be gathered by the follow-up team with the help of Behvarz in the near future.

\section{Assessment of the Pain Severity}

Assessment of pain is providing by McGill questionnaire [14). This tool has three categories of terms representing sensory, affective and evaluative records used to clarify pain experience subjectively. It gives scores to provide scales [14].

By the using Persian version of McGill pain inventory [15-19], translated and validated by
Khosravi et al. [17, 20], the pain severity is evaluated by trained anesthesiology nurses.

\section{Oswestry Questioner}

An outcome measure of functionality is used to assess the effect of LBP during the life. Oswestry is the gold standard of functional outcome tool for LBP patients by quantification scoring called Oswestry disability index (ODI). It interpretation clarifies the extent of functionality is affected by the pain in daily life $[16,21]$. For using this inventory, the transcultural validated Iranian translation is administered to all LBP suffering individuals [22].

These two types of questionnaire which especially were related to LBP were filled just for a patient that their answer to the screening question about LBP was "Yes."

The other important item which is recorded is interventions done previously for LBP as surgery or pharmacotherapy. Any diagnostic document including radiologic approaches will also be recorded.

\section{Follow-Up}

A15 year follow-up of LBP patients is planned in this cohort study. Incompatibility with Fasa cohort follow-up, all LBP patients are enrolled in a yearly follow-up by telephone calls. Besides other interested health events, the outcomes related to LBP is questioned. If any outcome is found out, the patient is invited to be visited by the pain/ anesthesiology specialist. The event is recorded and all essential data are included. The steps mentioned in the "LBP Evaluation section "is repeated for the patient. For those patients in the follow-up phase of Fasa cohort study who are new LBP cases, new registration in LBP cohort is done. They will be invited to fulfill all the "LBP Evaluation section." From then on, these newly enrolled cases will be under follow-up similar to older LBP patients.

\section{Discussion}

LBP studies in Iran are developing rapidly, both quantitatively and qualitatively in recent years. The problem is lacking large- scale longitudinal studies to provide more validated 
evidence- based data to help policy makers in planning better for this important health events.

Studies from developed countries on LBP have reported high point prevalence, one-year prevalence and increasingly high lifetime prevalence [23, 24]. Nonspecific mechanical LBP is the fifth most common reason for all physician visits in the United States, and the second most common symptomatic reason $[25,26]$.

According to the health economic surveys carried out in developed countries, LBP is also very costly $[27,28]$. Additionally, the days lost from work due to LBP increases the burdens. It has been suggested that LBP is the most common cause of activity limitations in people under the age of 45 [29].

Despite the great importance of LBP among non-communicable diseases, a comprehensive study regarding this disease has not been done in Iran.

Due to the high cost of LBP treatment and the high incidence of back surgery; preparing guidelines for an approach to the non-specific LBP seems necessary.

The cohort study is the best way to the Minis- try of Health to collect the necessary information required for policy making in this field. This study will help to provide some information about LBP in our area to establish a better management of the disease.

Moreover, this study will provide many opportunities for clinical trials in this field, and we are going to do interventional research in the cohort in future.

\section{Ethics approval and consent to participate}

This study follows the recommendations by Iranian national guidelines for ethics in research and is in accordance with Helsinki Declaration. The study protocol has been approved by the regional and national research ethics committees of Fasa University of Medical Sciences, (reference number: IR.FUMS. REC.1395.100). A written informed consent has been signed by all the participants after an agreement to participate in this study. The participants' data is confidential, and they are free to leave the study at any stage.

\section{Conflict of Interest}

None.

\section{References}

1. Frymoyer JW, Pope MH, Clements JH, Wilder DG, MacPherson B, Ashikaga T. Risk factors in low-back pain. An epidemiological survey. $\mathrm{J}$ Bone Joint Surg Am. 1983;65(2):213-8.

2. Garg A, Hegmann KT, Moore JS, Kapellusch J, Thiese MS, Boda S, et al. Study protocol title: a prospective cohort study of low back pain. BMC Musculoskelet Disord. 2013;14:84.

3. Stewart Williams J, Ng N, Peltzer K, Yawson A, Biritwum R, Maximova T, et al. Risk Factors and Disability Associated with Low Back Pain in Older Adults in Low- and Middle-Income Countries. Results from the WHO Study on Global AGEing and Adult Health (SAGE). PLoS One. 2015;10(6):e0127880.

4. Noormohammadpour P, Mansournia MA, Koohpayehzadeh J, Asgari F, Rostami M, Rafei A, et al. Prevalence of Chronic Neck Pain, Low Back Pain and Knee Pain and their Related Factors in Community-dwelling Adults in Iran: A Population-based National Study. Clin J Pain.
2016.

5. Mousavi SJ, Akbari ME, Mehdian H, Mobini B, Montazeri A, Akbarnia B, et al. Low back pain in Iran: a growing need to adapt and implement evidence-based practice in developing countries. Spine (Phila Pa 1976). 2011;36(10):E638-46.

6. Viniol A, Jegan N, Leonhardt C, Strauch K, Brugger M, Barth J, et al. Study protocol: Transition from localized low back pain to chronic widespread pain in general practice: identification of risk factors, preventive factors and key elements for treatment--a cohort study. BMC Musculoskelet Disord. 2012;13:77.

7. Nielsen AM, Vach W, Kent P, Hestbaek L, Kongsted A. Using existing questionnaires in latent class analysis: should we use summary scores or single items as input? A methodological study using a cohort of patients with low back pain. Clin Epidemiol. 2016;8:7389. 
8. Bradbury K, Al-Abbadey M, Carnes D, Dimitrov BD, Eardley S, Fawkes C, et al. Non-specific mechanisms in orthodox and CAM management of low back pain (MOCAM): theoretical framework and protocol for a prospective cohort study. BMJ Open. 2016;6(5):e012209.

9. Hendrick P, Milosavljevic S, Bell ML, Hale L, Hurley DA, McDonough SM, et al. Does physical activity change predict functional recovery in low back pain? Protocol for a prospective cohort study. BMC Musculoskelet Disord. 2009;10:136.

10. Melloh M, Aebli N, Elfering A, Roder C, Zweig T, Barz T, et al. Development of a screening tool predicting the transition from acute to chronic low back pain for patients in a GP setting: protocol of a multinational prospective cohort study. BMC Musculoskelet Disord. 2008;9:167.

11. Gore M, Sadosky A, Stacey BR, Tai KS, Leslie D. The burden of chronic low back pain: clinical comorbidities, treatment patterns, and health care costs in usual care settings. Spine (Phila Pa 1976). 2012;37(11):E668-77.

12. Farjam M, Bahrami H, Bahramali E, Jamshidi J, Askari A, Zakeri H, et al. A cohort study protocol to analyze the predisposing factors to common chronic non-communicable diseases in rural areas: Fasa Cohort Study. BMC Public Health. 2016;16(1):1090.

13. Soori H, Motlagh E. Iranian rural health workers (behvarz) and risk factors of childhood injury. East Mediterr Health J. 1999;5(4):684-9.

14. Melzack R. The McGill Pain Questionnaire: major properties and scoring methods. Pain. 1975;1(3):277-99.

15. 15. Dworkin RH, Turk DC, Trudeau JJ, Benson C, Biondi DM, Katz NP, et al. Validation of the Short-form McGill Pain Questionnaire-2 (SF-MPQ-2) in acute low back pain. J Pain. 2015;16(4):357-66.

16. Haas M, Nyiendo J. Diagnostic utility of the McGill Pain Questionnaire and the Oswestry Disability Questionnaire for classification of low back pain syndromes. J Manipulative Physiol Ther. 1992;15(2):90-8.

17. Kachooei AR, Ebrahimzadeh MH, ErfaniSayyar R, Salehi M, Salimi E, Razi S. Short Form-McGill Pain Questionnaire-2 (SFMPQ-2): A Cross-Cultural Adaptation and Validation Study of the Persian Version in Patients with Knee Osteoarthritis. Arch Bone Jt Surg. 2015;3(1):45-50.
18. Love A, Leboeuf C, Crisp TC. Chiropractic chronic low back pain sufferers and selfreport assessment methods. Part I. A reliability study of the Visual Analogue Scale, the Pain Drawing and the McGill Pain Questionnaire. J Manipulative Physiol Ther. 1989;12(1):21-5.

19. Prieto EJ, Hopson L, Bradley LA, Byrne M, Geisinger KF, Midax D, et al. The language of low back pain: factor structure of the McGill pain questionnaire. Pain. 1980;8(1):11-9.

20. Khosravi M, Sadighi S, Moradi S, K. Z. Persian-McGill pain questionnaire; translation, adaptation and reliability in cancer patients: a brief report. Tehran Univ Med J.71(1):53-8.

21. Fairbank JC, Pynsent PB. The Oswestry Disability Index. Spine (Phila Pa 1976). 2000;25(22):2940-52; discussion 52.

22. Baradaran A, Ebrahimzadeh MH, Birjandinejad A, Kachooei AR. Cross-Cultural Adaptation, Validation, and Reliability Testing of the Modified Oswestry Disability Questionnaire in Persian Population with Low Back Pain. Asian Spine J. 2016;10(2):215-9.

23. Walker BF. The prevalence of low back pain: a systematic review of the literature from 1966 to 1998. J Spinal Disord. 2000;13(3):205-17.

24. Walker BF. The prevalence of low back pain in Australian adults. A systematic review of the literature from 1966-1998. Asia Pac J Public Health. 1999;11(1):45-51.

25. Hart LG, Deyo RA, Cherkin DC. Physician office visits for low back pain. Frequency, clinical evaluation, and treatment patterns from a U.S. national survey. Spine (Phila Pa 1976). 1995;20(1):11-9.

26. Deyo RA, Mirza SK, Martin BI. Back pain prevalence and visit rates: estimates from U.S. national surveys, 2002. Spine (Phila Pa 1976). 2006;31(23):2724-7.

27. Luo X, Pietrobon R, Sun SX, Liu GG, Hey L. Estimates and patterns of direct health care expenditures among individuals with back pain in the United States. Spine (Phila Pa 1976). 2004;29(1):79-86.

28. 28. Straus BN. Chronic pain of spinal origin: the costs of intervention. Spine (Phila Pa 1976). 2002;27(22):2614-9; discussion 20.

29. 29. Andersson GB. Epidemiological features of chronic low-back pain. Lancet. 1999;354(9178):581-5. 